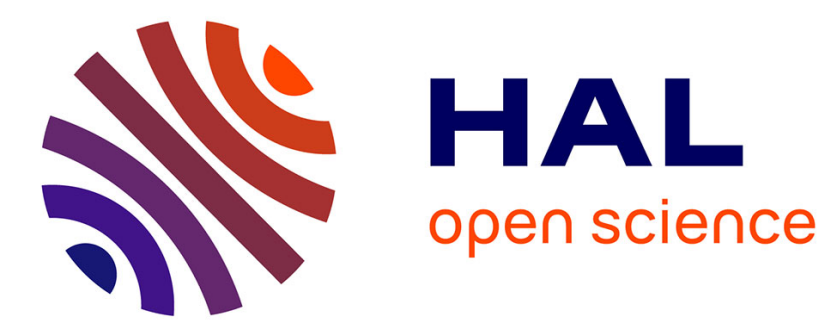

\title{
Les enjeux de la conciliation vie familiale - vie professionnelle en Europe
}

Blanche Le Bihan, Claude Martin

\section{To cite this version:}

Blanche Le Bihan, Claude Martin. Les enjeux de la conciliation vie familiale - vie professionnelle en Europe. presses de l'EHESP. Concilier vie familiale et vie professionnelle en Europe, Presses de l'EHESP, pp.5-34, 2008, Lien social et politiques, 978-2-85952-974-1. hal-00949117

\section{HAL Id: hal-00949117 https://hal.science/hal-00949117}

Submitted on 19 Feb 2014

HAL is a multi-disciplinary open access archive for the deposit and dissemination of scientific research documents, whether they are published or not. The documents may come from teaching and research institutions in France or abroad, or from public or private research centers.
L'archive ouverte pluridisciplinaire HAL, est destinée au dépôt et à la diffusion de documents scientifiques de niveau recherche, publiés ou non, émanant des établissements d'enseignement et de recherche français ou étrangers, des laboratoires publics ou privés. 


\title{
Les enjeux de la conciliation vie familiale - vie professionnelle en Europe
}

\author{
Blanche Le Bihan et Claude Martin
}

\section{Construire la conciliation vie familiale - vie professionnelle comme objet pour les sciences sociales}

L'expression «concilier vie familiale et vie professionnelle» est entrée dans le langage courant de l'action politique, notamment, nous le verrons, sous l'impulsion des institutions européennes et d'organisations internationales comme l'OCDE (2001; 2006). Elle désigne un objectif pour l'action publique en même temps qu'une série de mesures dont la vocation est en principe de faciliter la vie des citoyens. Il s'agit donc plutôt, pour l'heure, d'un énoncé normatif que d'une expression courante de la vie quotidienne. Du point de vue des parents, il est simplement question de faire face à diverses dimensions de leur existence, combinant dans des emplois du temps complexes leurs engagements, aspirations et contraintes familiales et professionnelles. Mais la «conciliation vie familiale - vie professionnelle » est-elle, et à quelles conditions, un objet pour les sciences sociales? Tel est le défi de cet ouvrage.

Nombre de spécialistes des études de genre refusent de recourir à cette expression du discours politique et lui préfèrent celles d'articulation entre vie familiale et vie professionnelle (Silvera, 2002). Leur résistance à son adoption réside dans le fait que cette expression contribue à masquer les inégalités de genre. Quoiqu'il en soit, le problème posé est celui de l'incompatibilité, du déséquilibre, des tensions et des cassures entre les temps sociaux : temps personnel, temps consacré à la vie familiale, temps de travail, temps de loisir, etc. Si nous reprenons néanmoins cette expression dans le cadre de cet ouvrage, c'est qu'elle désigne désormais une politique publique relativement explicite dans de nombreux pays de l'Union Européenne, dont la France (voir encadré plus bas). Même si la dénomination de ce secteur 
d'action publique continue d'évoluer - on parle ainsi dans les pays anglo-saxons de Work life balance - l'expression consacrée en français reste celle de conciliation, voire parfois de réconciliation.

De quoi s'agit-il donc et quelles sont les personnes concernées par ces politiques et mesures ? A priori, toute personne active peut avoir à concilier sa vie familiale et sa vie professionnelle, non seulement bien sûr les personnes inscrites sur le marché du travail, mais aussi les personnes en recherche d'emploi ; les personnes vivant en couple, avec ou sans enfant, mais aussi les jeunes travailleurs encore accueillis au domicile de leurs parents. Cette articulation entre vie familiale et vie professionnelle peut concerner non seulement des parents devant prendre soin de leurs enfants, quel que soit leur âge, mais aussi des adultes prenant soin d'un ou plusieurs de leurs ascendants devenus âgés et dépendants ou bien encore d'une personne handicapée ou malade, nécessitant une prise en charge quotidienne plus ou moins intense.

En réalité cependant, cette expression du discours politique (politics) et des politiques publiques (policies), vise sans le dire toujours explicitement des catégories beaucoup plus restreintes de citoyens : les parents de jeunes enfants ${ }^{1}$ occupant un emploi et devant faire face à leurs obligations de socialisation et de soins ; mais surtout, pour être plus précis encore, les mères de ces jeunes enfants, qui dans de nombreux pays assument en effet principalement cette charge de socialisation primaire et de care. Les mesures et politiques de conciliation sont donc surtout des politiques en direction des femmes, mais aussi en direction de la petite enfance (Martin, 2003 et 2004). On peut toutefois noter que les pays scandinaves envisagent au même niveau les besoins d'équilibre vie familiale - vie professionnelle pour les carers de personnes âgées dépendantes, ce qui n'est pas encore le cas en France, notamment.

Quels sont les principaux arguments des acteurs politiques pour défendre le développement de telles mesures et dispositions : promouvoir l'égalité hommes / femmes ; soutenir les ménages pour qu'ils actualisent leur désir d'enfant; permettre le renouvellement des générations et éviter ainsi le vieillissement global de la population; promouvoir l'emploi des femmes pour qu'elles contribuent à la production de richesses, à la croissance et à la pérennité des systèmes de protection sociale; lutter contre le chômage des femmes en développant des services de care conçus comme un «gisement d'emplois»; lutter contre la pauvreté infantile et les handicaps scolaires; contribuer à l'intégration des enfants issus de l'immigration; éviter le

\footnotetext{
${ }^{1}$. C'est-à-dire ceux qui ne sont pas encore entrés dans le système scolaire obligatoire : généralement les moins de six ans.
} 
stress des citoyennes et citoyens et tous ses effets négatifs en termes de santé ou de mal-être, mais aussi en termes de coûts sociaux?

Bien entendu, tous ces objectifs sont potentiellement présents dans les arguments avancés par les promoteurs de la conciliation vie familiale / vie professionnelle, avec des variations cependant, selon les lieux, les moments et les interlocuteurs. Le décryptage de ces logiques politiques n'est pas aisé, mais il est nécessaire pour comprendre les implicites du discours politique sur la conciliation, et montrer, par exemple, que l'objectif n'est peut-être pas tant d'égaliser la condition des femmes et des hommes ou de permettre aux mères, ou aux parents, de mener de front une carrière professionnelle et leurs rôles de parents, mais surtout de lutter contre le non renouvellement des générations (fécondité), ou bien encore de promouvoir le plein emploi ${ }^{2}$. Comme le souligne un récent rapport de l'OCDE : «Dans la mesure où la prospérité économique dépend du maintien d'un rapport emploi / population élevé, la volonté d'augmenter le taux d'activité des femmes est l'une des principales raisons pour lesquelles les pouvoirs publics souhaitent développer les services d'éducation et d'accueil des jeunes enfants »(OCDE, 2006, p. 12).

\section{La configuration française du débat sur la conciliation entre travail et vie de famille :}

L'agenda politique français est tout autant concerné par ces questions. La campagne pour les élections présidentielles de 2007 a révélé la place que pouvaient occuper ces enjeux domestiques dans les programmes politiques des principaux candidats. Le gouvernement de Dominique de Villepin avait également anticipé sur ces débats en confiant, d'une part au Centre d'analyse stratégique la mission de réfléchir aux conditions de mise en œuvre d'un « service public de la petite enfance » (CAS, 2007) et, d'autre part, en demandant à la députée Valérie Pécresse de lui remettre un rapport sur l'articulation vie familiale et vie professionnelle (Pécresse, 2007).

Ce dernier rapport reprend un certain nombre des arguments précédemment évoqués, tout en s'appuyant sur les mesures et «bonnes pratiques » développées par tel ou tel Etat membre de l'Union Européenne. Sans pouvoir prétendre que l'ordre de présentation de ces arguments constitue une véritable hiérarchie des priorités, on peut cependant les restituer tels qu'ils apparaissent dans le premier chapitre. L'articulation entre vies familiale et professionnelle est d'abord, selon ce rapport, un enjeu pour «la vitalité démographique d'une société, via la réalisation du désir d'enfants» (p. 15). C'est ensuite un "élément essentiel de la politique d'égalité entre les hommes et les femmes » (p.16), notamment en matière de partage du travail domestique et de care. C'est, également une politique qui a des conséquences sur le niveau d'emploi des femmes, lui-même «facteur de richesse collective» (p. 18). Quatrièmement, cette politique «joue un rôle important dans la création d'emploi. En effet, la solvabilisation de la demande de services de garde d'enfants contribue à développer un secteur d'activité considéré comme un gisement d'emplois accessibles aux personnes peu qualifiées (assistantes maternelles, aides éducatrices...) » (p. 20). C’est aussi un « outil de cohésion sociale » (p. 20)

\footnotetext{
${ }^{2}$. A moins qu'il ne s'agisse ou, comme le suggère Robert Castel, de promouvoir « la pleine activité » (Castel ; 2006) qui combine bien souvent flexibilité et précarité.
} 
permettant de lutter contre la pauvreté et la précarité des mères les plus modestes, et notamment celles qui du fait d'une rupture conjugale ou d'un veuvage précoce se retrouvent seules à élever leurs enfants. Enfin, une politique d'articulation vie familiale - vie professionnelle doit « offrir un libre choix aux familles » (p. 21).

Une fois encore, il est difficile de véritablement identifier les objectifs principaux, tant chacun d'entre eux semble étroitement imbriqué avec les autres. Seule une analyse détaillée des options choisies, des mesures adoptées, des arbitrages proposés, serait susceptible de nous permettre d'aller plus loin. Mais il est évident que les enjeux d'emploi sont particulièrement prégnants dans le cas français et que le souci de promouvoir l'emploi des femmes, en particulier le retour à l'emploi des femmes bénéficiaires de minima sociaux, la mise au travail des femmes à faible niveau de qualification, constituent des objectifs de premier ordre.

Parce qu'il s'agit principalement de politiques publiques, d'une part, et de conduites sociales, d'autre part, la conciliation vie familiale - vie professionnelle n'est pas un objet construit pour les sciences sociales. La difficulté principale réside précisément dans le fait de devoir rapprocher, combiner et faire dialoguer des travaux apparemment très éloignés les uns des autres, enracinés dans des disciplines ou dans des secteurs de spécialité différents. Notre projet est de contribuer à ce dialogue et au dépassement des frontières de spécialisation. Il est aussi de tirer le maximum de profits de la comparaison internationale, et ici surtout de la comparaison européenne. En effet, ce thème est beaucoup plus construit à l'étranger qu'il ne l'est en France et nous avons beaucoup à apprendre de ce débat européen, mais aussi nordaméricain. C'est la raison pour laquelle nous avons fait le choix de mettre en quelque sorte la situation française en regard de ce qui se passe à l'étranger, en Europe principalement, mais aussi au Québec.

Quels sont les angles possibles pour aborder la conciliation vie familiale - vie professionnelle ? On peut, comme nous l'avons déjà souligné, l'approcher sous l'angle de l'analyse des politiques publiques et, par exemple, retracer le genèse de ces législations et mesures, décrypter les objectifs avancés par tel ou tel Etat ou groupes d'acteurs, par telle ou telle institution (Union Européenne, par exemple); étudier les différentes modalités, techniques et dispositifs de conciliation. Le matériau du chercheur consiste alors en textes réglementaires, législations, règlements. Il peut privilégier, soit la construction de ces politiques au travers des rapports officiels, des débats parlementaires, des législations et règlements, soit leur mise en œuvre au travers des pratiques et discours des opérateurs nationaux et locaux. Il peut encore aborder ces dispositifs sous l'angle de leurs performances et résultats, et donc en termes évaluatifs. La conciliation est dans cette perspective d'abord et 
avant tout une politique, qui a ses objectifs, ses arguments, ses idées, ses promoteurs, ses opérateurs ; une politique qui peut être évaluée, suivie et comparée.

D'autres chercheurs abordent cette question sous l'angle d'une sociologie du travail et des professions, en s'intéressant à la manière dont chaque secteur d'activité est susceptible de poser le problème de l'articulation des temps de vie, en fonction de moult facteurs : la stabilité ou au contraire la précarité des emplois du secteur; la nature des horaires de travail plus ou moins standardisés, le degré de féminisation d'un secteur d'activité ou d'une profession, l'évolution des formes de management, le recours plus ou moins intense à la flexibilité, etc. L'angle impose alors des études empiriques poussées par secteur d'activité, car leur organisation, leur découpage et stratification, mais aussi leur management différent de manière importante. Pour ne prendre qu'un exemple, le secteur hospitalier impose aux professionnels des contraintes susceptibles de compliquer l'équilibre entre travail et vie familiale, et ce de manière variable selon les professions concernées, leur position dans la hiérarchie du système, etc. Seule une analyse approfondie de ces lieux d'exercice professionnels et secteurs est en mesure de répondre à la question de l'équilibre vie familiale - vie professionnelle.

Mais on peut aussi aborder la conciliation sous l'angle des pratiques sociales au sein des ménages et considérer que cet objet nécessite avant tout une étude empirique des manières de faire des individus, de leurs conduites, négociations et arrangements concrets. Comment en somme les acteurs font-ils face à cet ensemble complexe de rôles sociaux au quotidien, que recouvrent à la fois leur identité au travail et leur rôle de parent, de père ou de mère ? Cette sociologie de la conciliation en pratiques a recours à un tout autre matériau qui privilégie le point de vue des individus, mais aussi les arbitrages au niveau des ménages et des réseaux informels. Cette approche nécessite de tirer profit en même temps des apports de la sociologie de la famille et de l'analyse des rôles sexués. La question des inégalités sociales et des inégalités de genre est particulièrement importante de ce point de vue, car il est question de la contribution de chacun à la résolution des tensions engendrées par le cumul des tâches et des fonctions.

Dans cet ouvrage, nous proposons d'aborder l'objet sous ces divers angles successivement afin à la fois d'en montrer la complexité et la complémentarité ${ }^{3}$.

\footnotetext{
${ }^{3}$. La première partie adopte plutôt l'angle des politiques publiques. La seconde celui de l'analyse des idées et paradigmes ; la troisième, l'angle de la sociologie du travail et des secteurs d'emploi ; la quatrième, l'analyse des temps des familles et la dernière partie, l'analyse des arrangements de garde des enfants.
} 


\section{La conciliation vie familiale - vie professionnelle : un objectif pour les institutions européennes}

La conciliation vie familiale - vie professionnelle constitue manifestement pour les institutions européennes une réponse majeure aux nouveaux défis sociaux, économiques et démographiques que connaît l'Europe. Fortement investi par l'Union Européenne au début des années 1990, elle renvoie à une pluralité d'enjeux : celui de la croissance économique, de l'emploi, mais aussi de l'égalité hommes - femmes sur le marché du travail et dans le domaine du travail non rémunéré, à savoir les tâches de soin ou de care (la prise en charge des enfants et des personnes âgées dépendantes) et les tâches domestiques.

Comme le rappelle Jane Lewis (voir son chapitre dans cet ouvrage), la question de la conciliation vie familiale - vie professionnelle est historiquement liée à celle de l'égalité des hommes et des femmes. Signé en 1957, le Traité de Rome fait de l'égalité de rémunération entre les hommes et les femmes (article 119) une priorité de l'Union Européenne. L'enjeu est alors exclusivement économique; il s'agit de créer « des conditions égales pour tous », afin de garantir une concurrence équitable. La préoccupation des Etats membres est avant tout de poursuivre l'objectif d'un marché économique intérieur en Europe.

Initialement limitée à la question des salaires, l'égalité hommes - femmes s'étend progressivement à l'ensemble du marché du travail, avec l'adoption de différentes directives dans la deuxième moitié des années 1970 : la première (directive 75/117/CEE) décline le principe de l'égalité des rémunérations entre les travailleurs masculins et les travailleurs féminins ; la deuxième concerne de manière plus globale l'égalité d'accès à l'emploi, à la formation, à la promotion professionnelle et les conditions de travail (directive 76/207/CEE). Certes plus étendue que dans les premières années, les politiques en faveur de l'égalité hommes - femmes restent cependant limitées à la sphère professionnelle. Cette définition restrictive a été vivement critiquée par la littérature féministe et dénoncée par les mouvements de femmes, car elle exclut les différences entre hommes et femmes dans la sphère privée. En effet, tout ce qui ne relève pas directement des conditions du marché du travail reste en dehors du champ d'intervention publique de l'Union Européenne.

Il faut attendre la fin des années 1980 pour que cette idée d'égalité s'étende au-delà du marché du travail et interroge de manière globale la position des hommes et des femmes. En effet, les inégalités ne se développent pas uniquement sur le marché du travail, mais aussi 
dans la sphère privée. Elles concernent également la répartition des tâches domestiques et de care entre les hommes et les femmes et leur réduction suppose un changement d'attitude des hommes. Cet intérêt pour le travail de care au sein des familles, le lien établi entre la division de ce travail non rémunéré et le niveau de participation des femmes au marché du travail, et l'interrogation sur la nécessité pour les hommes de modifier leur comportement constituent une avancée majeure du traitement des inégalités sociales (Hantrais, 2000). Elle se traduit par le recours à la notion de conciliation vie familiale - vie professionnelle, qui s'impose au début des années 1990 comme le moyen de permettre aux parents de jeunes enfants de faire face à leurs obligations professionnelles et à leurs responsabilités familiales. Sphère professionnelle et sphère privée sont dès lors pensées ensemble, l'investissement dans le travail non rémunéré ayant des conséquences directes sur la capacité des parents à travailler.

La notion de conciliation est introduite dès 1989 dans une communication sur les politiques familiales (Communication 1989, 363) ${ }^{4}$, qui reconnaît le double investissement des femmes dans l'emploi et dans la vie familiale et s'interroge sur les moyens d'assurer un partage plus équilibré des responsabilités familiales entre les hommes et les femmes, et de «concilier vie professionnelle et vie familiale » (Jönsson et Perrier, 2007).

Cette nouvelle orientation politique se traduit par deux mesures principales: la Recommandation de 1992 (Conseil, 1992) concernant la garde des enfants et la directive de 1996 sur le congé parental. Instrument non contraignant, la Recommandation de 1992 encourage les Etats membres à développer des initiatives «permettant aux hommes et aux femmes de concilier leurs responsabilités professionnelles, familiales et de prise en charge d'un ou plusieurs enfants ». Quatre types d'action sont proposées aux Etats membres afin de faciliter cette conciliation vie familiale - vie professionnelle : le développement de services de garde pour les enfants dont les parents travaillent; l'élaboration de politiques d'entreprises tenant compte des besoins des familles; la participation des hommes à la prise en charge des enfants et l'élaboration de congés donnant aux deux parents la possibilité de s'occuper de leurs enfants. Pour la première fois, l'Union européenne propose une mesure, certes souple et uniquement sous la forme de recommandation, allant au-delà du marché du travail et englobant la sphère privée. Par ailleurs, elle s'affiche comme neutre du point de vue du genre, puisqu'elle concerne les hommes comme les femmes. Elle témoigne d'une évolution des frontières de l'action publique entre la sphère publique du marché du travail et la sphère

\footnotetext{
${ }^{4}$ Cette notion de conciliation apparaissait dans le Programme communautaire d'action sociale de 1974, mais elle n’avait pas été reprise ensuite.
} 
privée de la vie familiale (Stratigaki, 2004 ; Jönsson et Perrier, 2007) et d'une reconnaissance des interrelations qui existent entre les deux. Il est désormais légitime pour les Etats d'intervenir dans la sphère privée, en développant des mesures visant à réduire les inégalités hommes - femmes en matière de travail non rémunéré.

Contrairement à la recommandation de 1992, la directive de 1996 sur le congé parental a une dimension législative contraignante. Elle instaure le droit individuel à un congé minimum de trois mois, accordé aux hommes et aux femmes, suite à la naissance ou l'adoption d'un enfant. Dans un souci d'égalité hommes - femmes, elle stipule par ailleurs que ce congé n'est pas transférable d'un parent à l'autre. L'application de cette mesure reste cependant très souple, puisqu'il revient aux Etats membres de définir les conditions d'accès à ce congé, mais aussi et surtout de décider de sa rémunération ou non et à quelle hauteur. Comme le rappelle Jane Lewis (dans cet ouvrage), cette absence de rémunération minimale a limité fortement les chances de voir les pères prendre ce congé. En définitive, les règles ainsi instaurées ne sont pas très incitatives et laissent aux Etats membres une importante marge de manœuvre.

L'analyse des textes communautaires déclinant les orientations politiques de l'Union européenne depuis la fin des années 1990 semble aller dans le sens de cette conciliation vie familiale - vie professionnelle (Silvera, 2002). La Stratégie européenne pour l'emploi adoptée en 1997 souligne ainsi la nécessité de renforcer l'égalité hommes - femmes : «Les Etats membres doivent s'attaquer à la discrimination entre hommes et femmes, prendre les mesures appropriées pour permettre aux hommes et aux femmes de concilier vie professionnelle et vie familiale et faciliter la réintégration des hommes et des femmes dans la vie active » (résolution du conseil européen, 1997). De même, la Résolution du Conseil des ministres de l'Emploi souligne à nouveau l'importance d'une participation équilibrée des hommes et des femmes à la vie familiale et professionnelle : «Le principe de l'égalité entre les hommes et les femmes impose de compenser le désavantage des femmes en ce qui concerne les conditions d'accès et de participation au marché du travail et le désavantage des hommes pour ce qui est des conditions de participation à la vie familiale, désavantages résultant de pratiques sociales qui font toujours considérer le travail non rémunéré accompli dans l'intérêt de la famille comme une responsabilité principale des femmes et le travail rémunéré dans la sphère économique comme une responsabilité principale des hommes »(Conseil, 2000). On peut également mentionner le cinquième programme d'action en faveur de l'égalité des chances (2001-2005) ou encore le Pacte européen pour l'égalité entre les hommes et les femmes de 
2006 (Conseil européen, 23-24 mars 2006), qui réaffirment la nécessité d'atteindre l'égalité dans la vie économique comme dans la vie sociale.

Ces directives, recommandations, programmes ou orientations en faveur de la conciliation vie familiale - vie professionnelle traduisent-elles une intégration réelle du principe d'égalité hommes femmes par l'Union européenne ? Comme le montre Jane Lewis dans son analyse de la politique européenne en matière d'égalité hommes - femmes, la question est en fait celle du sens des politiques menées. Quels sont précisément les objectifs poursuivis par la mise en œuvre de telles mesures ? Quelles logiques politiques sont ainsi affirmées et quelle logique principale parvient finalement à s'imposer ?

L'analyse de ces politiques de conciliation dans la durée montre en fait le glissement progressif d'un objectif d'égalité entre les hommes et les femmes et de partage des responsabilités dans la sphère professionnelle comme dans la sphère privée, vers un objectif économique, centré sur l'emploi et le développement de la flexibilité au travail (Stratigaki, 2004). Selon Jane Lewis, la fin des années 1990 marque ainsi une profonde évolution de la façon dont sont définies les politiques de conciliation. Leur inscription dans les lignes directrices de la Stratégie européenne pour l'emploi traduit l'accent mis sur le lien avec l'emploi, notamment pour les femmes. Il ne s'agit donc plus tant de promouvoir l'égalité hommes - femmes en modifiant l'attitude des hommes et en les impliquant beaucoup plus dans les activités domestiques et de care, mais plutôt de développer l'offre de garde, afin de libérer du temps pour les femmes et leur permettre de travailler tout en ayant des enfants. Le conseil de Lisbonne (2000) fixe ainsi l'objectif d'atteindre d'ici à 2010 dans tous les Etats membres un taux d'emploi des femmes de $60 \%$ et deux ans plus tard, le conseil de Barcelone pose celui d'accueillir $90 \%$ des enfants de plus de 3 ans et $33 \%$ des enfants âgés de moins de 3 ans.

L'examen des politiques adoptées par l'Union Européenne révèle donc l'ambiguïté de l'objectif d'égalité hommes - femmes. Il pose la question du travail rémunéré et de l'égalité de traitement, mais également celle du travail non rémunéré et de la répartition des tâches domestiques et de care. L'évolution des mesures prises par l'Union européenne tend à montrer que la thématique de la conciliation, adoptée au début des années 1990, a peu à peu glissé vers une approche économique de cet enjeu d'égalité, centrée sur l'emploi. L'objectif de modification des comportements des hommes au sein de la sphère privée défendu par les féministes tend à devenir secondaire, par rapport à la logique exclusivement économique visant à permettre aux femmes comme aux hommes de participer au marché du travail. C'est 
d'ailleurs à partir du moment où elle a été fortement liée aux politiques d'emploi que la conciliation est devenue un enjeu central de l'agenda politique européen. Au bout du compte, les mesures de conciliation ne modifient pas l'inégale répartition des tâches entre les hommes et les femmes dans la sphère privée et, au contraire, peuvent contribuer à maintenir le modèle de la femme principale pourvoyeuse de soin (Lewis,1997 ; Stratigaki, 2004).

L'importance des enjeux macro-économiques conduit certains auteurs, comme Gøsta EspingAndersen (2008), à défendre précisément en termes économiques ces enjeux de conciliation et d'égalité. Partant du constat que « la modification de la place des femmes dans la société est la force de changement la plus révolutionnaire », cet auteur développe un argument auquel les décideurs européens pourraient bien être sensibles. Il montre ainsi que le développement d'une politique ambitieuse de services à la petite enfance est un véritable «investissement social » (voir aussi Esping-Andersen, 2002), susceptible de promouvoir en même temps fécondité et croissance. En donnant la possibilité aux femmes d'accéder au travail rémunéré, l'externalisation des tâches de care vers des services de qualité, permet à la fois aux ménages d'actualiser leur désir d'enfants (qui correspond à un peu plus de deux enfants par couple dans tous les pays européens), d'améliorer le revenu des ménages et ainsi d'éviter la pauvreté pour un nombre significatif d'enfants, mais aussi d'accroître les recettes fiscales.

Ce « retour sur investissement » doit cependant, selon lui, aussi tenir compte de trois variables essentielles: premièrement, une politique de conciliation doit «renforcer le pouvoir de négociation de la femme au sein de la famille »(Esping-Andersen, 2008, p. 35), ce qui suppose de «féminiser les parcours de vie masculins » en les incitant à assumer des tâches domestiques et de soins mais aussi d'améliorer le niveau de rémunérations des femmes ; deuxièmement, les emplois stables facilitent la décision d'avoir des enfants ${ }^{5}$, ce qui impose de promouvoir des emplois stables pour les femmes et non des emplois précaires; troisièmement, les parents, - et notamment les pères ou les grands parents - ont d'autant plus de chance d'assumer un rôle de care que l'intensité de ce travail est réduite (en nombre d'heures consacrées par jour). En d'autres termes, lorsque l'intensité du travail de care est trop élevée, il conduit à l'épuisement de ceux qui l'assument. Le fait de formuler les enjeux de conciliation en termes économiques, comme le fait Esping-Andersen, a sans doute pour vertu de rendre ses arguments plus recevables par les décideurs politiques. Mais il montre d'une autre manière cependant le poids de cette logique économique aux dépens de la défense

\footnotetext{
5 . «Les femmes bénéficiant de contrats de travail stables ont deux fois plus de chances de mettre un enfant au monde que les femmes munies d'un contrat à durée déterminée. » (op. cit., p. 33-34).
} 
d'une citoyenneté sociale, quel que soit le genre des citoyens. Ce type de présentation des enjeux de conciliation se retrouve également dans les travaux menés par l'OCDE. Rianne Mahon (voir son chapitre dans cet ouvrage) montre ainsi comment cette organisation internationale est traversée par des tensions en termes de référentiels ou d'idées entre d'une part la position néo-libérale défendue au plan des politiques économiques et d'emploi et, d'autre part, une position de type «troisième voie», qui fait de la question de l'investissement social dans l'enfance un enjeu de premier ordre.

\section{La diversité des politiques nationales}

Même si les institutions européennes ont largement contribué à promouvoir ces politiques de conciliation, celles-ci ne s'inscrivent cependant pas dans un même contexte selon les Etats. Les variations nationales sont considérables, que ce soit en termes de niveau de fécondité, de position des femmes sur le marché du travail, ou en termes de mesures, de logiques politiques et de contextes institutionnels. Il va de soi que ces indicateurs jouent un rôle crucial pour développer tel ou tel argument, défendre telle ou telle mesure. Même si l'Union européenne contribue à façonner les politiques nationales, comme nous venons de le rappeler, celles-ci restent néanmoins profondément déterminées par la configuration et la hiérarchie des problèmes publics tels que construits par les gouvernements et les acteurs sociaux.

L'argument concernant les enjeux de renouvellement des générations et de déficit de fécondité sont évidemment très présents au plan européen. Seule la France se trouve depuis quelques années pratiquement au seuil de renouvellement des générations. Mais, comme l'indique le tableau 1, cette situation est assez exceptionnelle. Dix-sept des vingt-sept Etats membres ont actuellement un indice conjoncturel de fécondité inférieur à 1,5 enfant en moyenne par femme. Cinq pays seulement ont un indicateur supérieur à 1,8, dont les trois pays scandinaves, la France, l'Irlande et le Royaume-Uni. Le fait que le niveau de fécondité soit plus élevé dans des pays où les politiques en matière de services à la petite enfance sont particulièrement développées (comme en France ou en Scandinavie) a joué un rôle tout à fait déterminant pour faire évoluer la représentation courante consistant à considérer que la fécondité était plus élevée dans les pays où les femmes n'étaient pas inscrites sur le marché du travail. Cette vulgate a bientôt fait place à une autre interprétation qui avance au contraire que la fécondité dépend surtout du fait que les femmes puissent échapper au dilemme consistant à 
choisir entre faire carrière ou avoir des enfants. Sous cet angle, fécondité et conciliation vont donc de pair.

Tableau 1: Indice conjoncturel de fécondité dans les pays membres de l'Union européenne (source Eurostat)

\begin{tabular}{|c|c|c|c|c|}
\hline & 1997 & 2000 & 2003 & 2006 \\
\hline France & & $\mid 1.89$ & $\mid 1.89$ & 2.00 \\
\hline Irlande & $\mid 1.93$ & $\mid 1.89$ & $\mid 1.97$ & \begin{tabular}{l|l}
7 & 1.93 \\
\end{tabular} \\
\hline Suède & $\mid 1.52$ & $\mid 1.54$ & $\mid 1.71$ & 1.85 \\
\hline Finlande & $\mid 1.75$ & 1.73 & \begin{tabular}{|l|l|}
3 & 1.77 \\
\end{tabular} & 1.84 \\
\hline Royaume-Uni & 1.72 & $\mid 1.64$ & 1.71 & 1.84 \\
\hline Danemark & 1.76 & 1.78 & $\mid 1.76$ & 51.83 \\
\hline Belgique & $\mid 1.60$ & & & $: 1,72^{6}$ \\
\hline Pays-Bas & $\mid 1.56$ & 1.72 & $\mid 1.75$ & $\mid 1.70$ \\
\hline Luxembourg & $\mid 1.72$ & 1.76 & \begin{tabular}{|l|l|}
$\mid$ & 1.63 \\
\end{tabular} & 1.65 \\
\hline Estonie & 1.32 & 1.39 & 1.37 & 1.55 \\
\hline Chypre & 1.86 & 1.64 & 1.50 & \\
\hline \multicolumn{5}{|l|}{ Malte } \\
\hline Autriche & $\mid 1.39$ & 1.36 & $5 \mid 1.38$ & \\
\hline Grèce & $\mid 1,28$ & 1,26 & $5 \mid 1,28$ & \\
\hline Espagne & $\mid$\begin{tabular}{|l}
$\mid 1.17$ \\
1.
\end{tabular} & 1.23 & \begin{tabular}{|l|l|}
$\mid 1.31$ \\
$\mid$
\end{tabular} & \\
\hline Bulgarie & $\mid 1.09$ & 1.26 & $5 \mid 1.23$ & \\
\hline Portugal & 1.47 & 1.55 & $5 \mid 1.44$ & \\
\hline Lettonie & $\mid 1.14$ & 1.24 & $\mid 1.29$ & 35 \\
\hline Hongrie & $\mid 1.37$ & 1.32 & $\mid 1.27$ & .34 \\
\hline Italie & 1.21 & 1.26 & 1.29 & 1,34 \\
\hline République tchèque & $\mid$\begin{tabular}{|ll}
$\mid 1.17$ & -17
\end{tabular} & $\mid 1.14$ & $\mid 1.18$ & $\mid 1.3$ \\
\hline Allemagne & $\mid 1.37$ & 1.38 & \begin{tabular}{|l|l|}
$\mid$ & 1.34 \\
\end{tabular} & \\
\hline Lituanie & $\mid 1.47$ & 1.39 & 1.26 & \\
\hline Roumanie & 1.40 & 1.39 & 1.27 & 1.31 \\
\hline Slovénie & $\mid 1.25$ & 1.26 & $5 \mid 1.20$ & 1.31 \\
\hline Pologne & $\mid 1.52$ & 1.35 & $\mid 1.22$ & $\begin{array}{ll}2 & 1.27\end{array}$ \\
\hline Slovaquie & 1.43 & 1.29 & 1.20 & 1.24 \\
\hline
\end{tabular}

La situation est tout aussi contrastée en matière de niveau d'emploi des femmes (voir tableau 2). Si l'objectif fixé en 2000 au Conseil de Lisbonne est d'atteindre un taux d'emploi des femmes de $60 \%$ d'ici à 2010, chaque Etat membre ne part pas du même point. En 2006, 13 des 27 Etats avaient déjà franchi ce seuil.

Tableau 2: Taux d'emploi des femmes (source Eurostat)

\begin{tabular}{l|||c|c|c|c|}
\hline & 1997 & 2000 & 2003 & 2006 \\
\hline UE (27 pays) & 51.4 & 53.7 & 54.9 & 57.3 \\
\hline UE (25 pays) & 51.1 & 53.6 & 55.2 & 57.6 \\
\hline UE (15 pays) & 50.8 & 54.1 & 56.2 & 58.7 \\
\hline Union européenne & 50.8 & 54.1 & 56.2 & 57.6 \\
\hline Danemark & 69.1 & 71.6 & 70.5 & 73.4 \\
\hline
\end{tabular}

${ }^{6}$ En 2005.

${ }^{7}$ En 2005. 


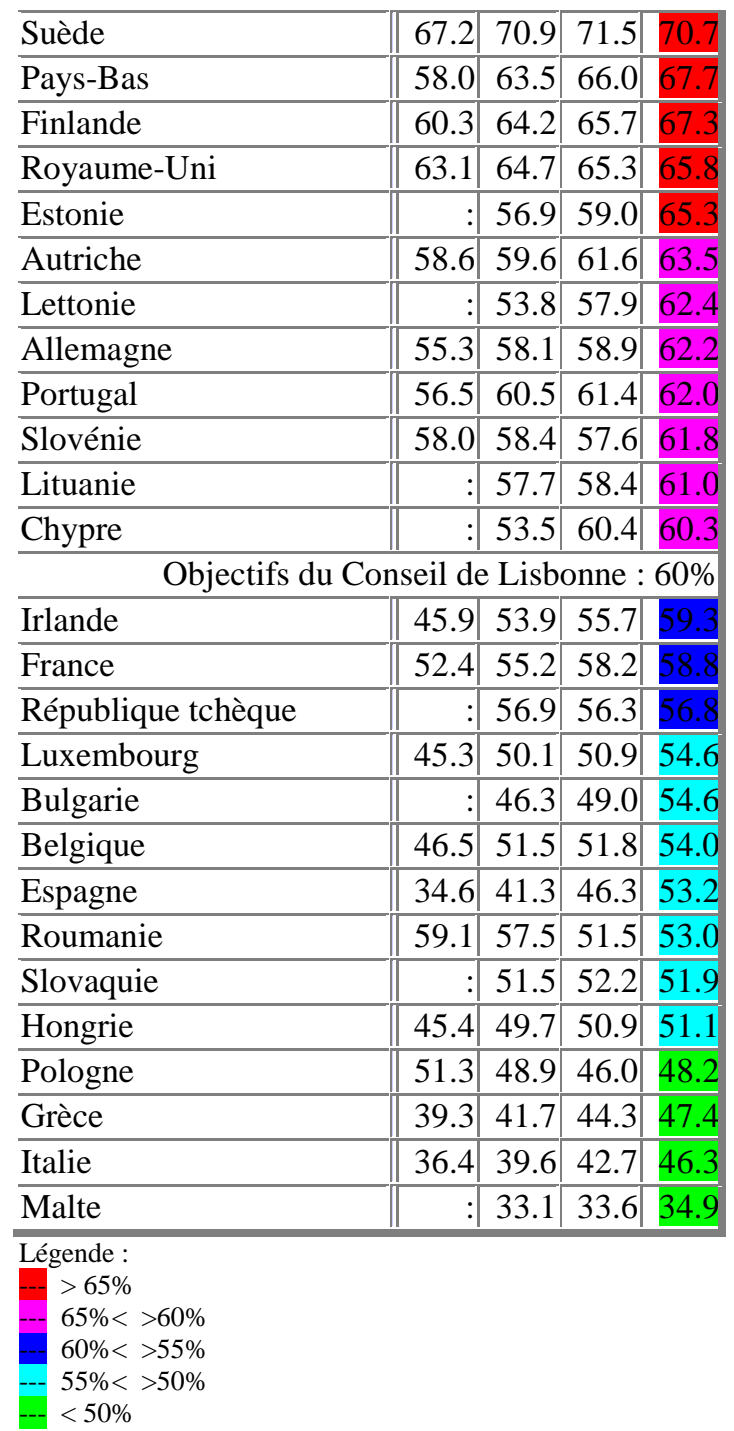

Le recours au travail à temps partiel est une des modalités de conciliation vie familiale - vie professionnelle, en particulier pour les femmes. Même si ce niveau de temps partiel féminin ne correspond pas toujours à une politique explicite d'emploi et, donc a fortiori, de conciliation, on constate qu'une fois encore, la situation est très contrastée en Europe (voir tableau 3). A l'exception du cas particulier des Pays-Bas où l'emploi à temps partiel est quasiment la norme pour les femmes (et conçu comme une politique de conciliation), cinq pays ont un taux supérieur à 40\%: Allemagne, Royaume-Uni, Belgique, Autriche et Suède.

Tableau 3 : Femmes ayant un emploi à temps partiel (\% de l'emploi total) (source :

Eurostat, 2008, http://epp.eurostat.ec.europa.eu/tgm/table.do?tab=table\&init=1\&plugin=0\&language=fr\&pcode=ccb22820)

\begin{tabular}{|l|l|l|l|l|}
\hline & 1997 & 2000 & 2003 & 2006 \\
\hline UE (27 pays) & 29.2 & 28.9 & 29.0 & 31.2 \\
\hline UE (25 pays) & 29.8 & 29.5 & 30.2 & 32.6 \\
\hline UE (15 pays) & 32.2 & 33.2 & 33.8 & 36.7 \\
\hline Zone euro & 29.5 & 31.3 & 31.5 & 35.2 \\
\hline Pays-Bas & 67.3 & 71.0 & 74.1 & 74.7 \\
\hline Allemagne & 35.3 & 37.9 & 40.8 & 45.6 \\
\hline Royaume-Uni & 44.6 & 44.3 & 44.0 & 42.6 \\
\hline
\end{tabular}




\begin{tabular}{|c|c|c|c|c|}
\hline Belgique & 32.4 & 37.4 & 39.1 & 41 \\
\hline Autriche & 28.5 & 32.2 & 36.0 & 40 \\
\hline Suède & \begin{tabular}{|l|}
34.7 \\
\end{tabular} & 32.3 & 35.5 & $\sqrt{11}$ \\
\hline Luxembourg & 21.0 & 25.1 & 30.7 & 36.2 \\
\hline Danemark & \begin{tabular}{|l|}
34.9 \\
\end{tabular} & 34.1 & 32.7 & 35.4 \\
\hline Irlande & 25.4 & 30.3 & 31.0 & \\
\hline France & 31.2 & 30.8 & 29.6 & 30.2 \\
\hline Italie & \begin{tabular}{|l|}
13.4 \\
\end{tabular} & 16.5 & 17.3 & 26.5 \\
\hline Espagne & 17.0 & 16.8 & 17.1 & 23.2 \\
\hline Malte & : & 15.5 & 21.3 & 21.8 \\
\hline Finlande & 15.3 & 17.0 & 17.7 & 19.2 \\
\hline Portugal & 16.6 & 16.4 & 16.9 & 15.8 \\
\hline Pologne & \begin{tabular}{|l|}
13.6 \\
\end{tabular} & 13.4 & 13.2 & 13.0 \\
\hline Chypre & : & 13.9 & 13.2 & 12.1 \\
\hline Lituanie & : & 11.1 & 11.8 & 12.0 \\
\hline Slovénie & $:$ & 7.8 & 7.5 & 11.6 \\
\hline Estonie & $:$ & 10.9 & 11.8 & 11.3 \\
\hline Grèce & 8.5 & 7.8 & 7.7 & 10.2 \\
\hline Roumanie & 17.5 & 18.6 & 12.2 & 9.8 \\
\hline République tchèque & $:$ & 9.3 & 8.5 & 8.7 \\
\hline Lettonie & $:$ & 12.8 & 12.7 & 8.3 \\
\hline Hongrie & 5.6 & 5.2 & 6.2 & 5.6 \\
\hline Slovaquie & : & 3.1 & 3.8 & 4.7 \\
\hline Bulgarie & : & $:$ & 2.6 & 2.5 \\
\hline \multicolumn{5}{|l|}{$\begin{array}{l}\text { Légende }: \\
\quad>40 \% \\
\quad 40 \%<\text { et }>30 \% \\
30 \%<\text { et }>20 \% \\
-\quad<20 \%\end{array}$} \\
\hline
\end{tabular}

Il faut également tenir compte du fait que cet emploi à temps partiel soit un «temps partiel long » (supérieur à 20 heures hebdomadaire $)^{8}$ ou court (inférieur à 20 heures) ${ }^{9}$. Là encore la situation varie sensiblement selon les pays.

A part le recours au temps partiel, voire aux modalités de régulation du temps de travail (qui peut être étatique ou négociée par branche d'activité ou par entreprise), les politiques de conciliation vie familiale - vie professionnelle s'appuie essentiellement sur deux mesures : le développement de services d'accueil des jeunes enfants donnant aux parents la possibilité de faire garder leurs enfants pendant leur temps de travail ; les congés parentaux, qui permettent aux parents de s'occuper eux-mêmes de leur enfant pendant un certain temps.

Le développement des modes d'accueil pour les jeunes enfants est présenté comme une réponse particulièrement adaptée à l'articulation entre vie familiale et vie professionnelle.

\footnotetext{
${ }^{8}$ Comme c'est le cas dans la majorité des pays de l'UE des 27 : Suède, France, Belgique, Luxembourg, Italie, Pologne, Roumanie, Hongrie, Lituanie, République Tchèque, Slovaquie, Lettonie, Estonie, Malte.

9 . Comme aux Pays-Bas, au Portugal, Espagne, Slovénie, Danemark, Royaume-Uni, Allemagne et Irlande. La Finlande, la Grèce, la Bulgarie, l'Autriche et Chypre ont une durée hebdomadaire de temps partiel d'environ 20 heures.
} 
C'est d'ailleurs, comme nous l'avons vu, l'objectif affiché de la politique européenne en matière de conciliation. Cependant, comme le constate certains auteurs dans leur contribution à cet ouvrage (Kremer, 2007) les données disponibles sur les systèmes mis en place dans les différents pays sont loin d'être homogènes. Il faut tenir compte de l'âge des enfants, mais aussi de la qualité et de l'accessibilité des services proposés. En outre l'articulation de cette prise en charge des jeunes enfants avec le système scolaire varie d'un pays à l'autre. L'existence en France par exemple d'un système scolaire étendu, avec une prise en charge gratuite des enfants dès l'âge de 3 ans, voire de 2 ans, est un élément déterminant de l'offre de services proposée.

Il est particulièrement difficile de recueillir des données comparables sur les modes d'accueil des enfants de moins de 3 ans. Comme le constate la littérature sur le sujet (Silvera, 2002 ; Périvier, 2004), les progrès accomplis en matière de mode d'accueil concernent surtout les enfants de 3 à 6 ans. Le taux de couverture des 0-3 ans reste quant à lui très faible. Les arrangements de garde mis en place sont complexes, associant offre publique, mais aussi services privés et ressources informelles difficiles à recenser. En outre, l'organisation souvent territoriale des modes de garde public introduit de fortes disparités locales et il est difficile d'avoir une vision globale de l'offre de garde.

De manière générale, trois possibilités sont offertes aux parents pour faire garder leurs jeunes enfants : les modes de garde collectifs (crèches, centres de loisir, day care centre...), les assistantes maternelles ou nourrices qui gardent les enfants chez elles, enfin, la garde à domicile. L'analyse porte plus spécifiquement sur la première de ces solutions. Il est dès lors possible de distinguer les pays où l'offre publique est développée comme les pays scandinaves, la France ou la Belgique et les pays où au contraire, l'offre est insuffisante comme en Allemagne, en Autriche, aux Pays Bas, au Royaume-Uni, en Irlande, et dans les pays d'Europe du Sud. Mais comme le montre la littérature, l'analyse ne peut se limiter à une description de l'offre seule. Cette dernière renvoie à des normes sociales spécifiques et au rôle donné à l'Etat en matière de politique familiale (Martin, 2002). On peut ainsi distinguer les pays nordiques, où l'intervention publique est jugée essentielle et les structures d'accueil nombreuses, répondant au souci affiché d'égalité entre les citoyens. Les structures de prise en charge des jeunes enfants y sont donc très développées. Les pays méditerranéens constituent un deuxième groupe, où les solidarités familiales restent le pivot de la conciliation vie familiale - vie professionnelle ; la France et la Belgique un troisième, avec, comme dans les pays scandinaves un investissement public important dans le secteur de la petite enfance, mais 
pour des raisons différentes. L'objectif nataliste y est en effet fondamental. L'Allemagne, l'Autriche et les Pays-Bas proposent tous les trois très peu de services publics, mais cette organisation correspond à l'importance accordée par ailleurs à l'investissement des parents dans la socialisation du jeune enfant. Le Royaume Uni et l'Irlande constituent un dernier groupe, caractérisé par une faible intervention de l'Etat dans la sphère privée (Périvier, 2004).

Pour comprendre les politiques de conciliation vie familiale - vie professionnelle dans les différents pays, il faut prendre en compte le deuxième instrument d'action dont disposent les gouvernements: les congés parentaux. L'objectif est alors de permettre aux parents d'interrompre leur activité professionnelle et de leur donner du temps pour s'occuper de leurs enfants. Les types de congés sont multiples : le congé maternité, le congé paternité, le congé parental. Les caractéristiques de ces congés varient d'un pays à l'autre, de même que l'articulation entre les congés existants Deux enjeux émergent plus particulièrement : la durée du congé et sa rémunération

Depuis le milieu des années 1990, les congés maternité ont été augmentés en Belgique, au Danemark, en Grèce, en Irlande, en Islande, au Luxembourg, aux Pays Bas, au Portugal et au Royaume-Uni. La durée reste très variable d'un pays à l'autre, allant de 14 semaines en Allemagne (durée minimale définie dans la directive de la commission européenne) à environ 6 mois en Hongrie, en Irlande, au Royaume Uni ou en Slovaquie. Mais pour bien appréhender les réalités nationales, il faut tenir compte du congé parental, qui prolonge souvent le congé maternité ou l'englobe (Math et Meilland, 2004). Ainsi, les cas de la Suède et de la Norvège, où il n'existe pas de congé maternité en tant que tel, mais un congé parental, de 480 jours pour le premier et de 52 semaines pour le second.

Tableau 4 : Durée et rémunération du congé légal de maternité (source : Math et Meilland, 2004, p.117)

\begin{tabular}{|c|c|c|c|c|}
\hline \multirow{2}{*}{\multicolumn{2}{|c|}{ Fin 2003}} & \multicolumn{3}{|c|}{ Niveau de rémunération (compensation en \% du salaire) } \\
\hline & & Moins de $80 \%$ & Intermédiaire & $100 \%$ \\
\hline \multirow{4}{*}{$\begin{array}{r}\mathrm{D} \\
\text { urée }\end{array}$} & $\begin{array}{c}14-15 \\
\text { semaines }\end{array}$ & Belgique & & $\begin{array}{l}\text { Allemagne } \\
\text { Slovénie }\end{array}$ \\
\hline & $\begin{array}{c}16-18 \\
\text { semaines }\end{array}$ & Finlande & Danemark & $\begin{array}{l}\text { Autriche } \\
\text { France } \\
\text { Grèce } \\
\text { Luxembourg } \\
\text { Pays-Bas } \\
\text { Pologne } \\
\text { Portugal } \\
\text { Espagne }\end{array}$ \\
\hline & $\begin{array}{c}21 \\
\text { semaines }\end{array}$ & & Italie & \\
\hline & \begin{tabular}{l}
\multicolumn{1}{c}{24} \\
semaines \\
et +
\end{tabular} & $\begin{array}{l}\text { Hongrie } \\
\text { Irlande } \\
\text { Royaume-Uni* }\end{array}$ & $\begin{array}{l}\text { Royaume-Uni** } \\
\text { Slovaquie } \\
\text { Suède } \\
\text { *** }\end{array}$ & Norvège $* * *$ \\
\hline
\end{tabular}

*Pour les 20 premières semaines consécutives aux 6 premières

**Pour les 6 premières semaines 
*** Pas de congé maternité spécifique.

La durée du congé parental varie d'un pays à l'autre. Elle va en effet du minimum de 13 semaines au Royaume-Uni et en Belgique à 3 ans en France, en Allemagne, en Hongrie, en Pologne, en Espagne et en Slovaquie (Math et Meilland, 2004a). Cette alternative entre congés courts et congés longs est un enjeu essentiel de la politique de conciliation menée. En effet, un congé court a l'avantage de ne pas introduire de rupture trop longue d'activité professionnelle et par là même de faciliter le retour sur le marché du travail. Reste qu'il suppose une organisation publique et/ou privée permettant aux familles de faire face à leurs obligations professionnelles et à leurs responsabilités familiales.

Mais cette question de la durée doit être pensée en lien avec celle de la rémunération du congé. Pour ce qui concerne le congé maternité, elle existe aujourd'hui dans l'ensemble des pays, sous forme d'une compensation proportionnelle au revenu d'activité, qui varie d'un pays à l'autre (tableau 4). Elle est ainsi plus faible au Danemark, en Italie, en Slovaquie, en Suède et au Royaume-Uni. Elle est plafonnée au Danemark, en Slovaquie et en Suède. Tout dépend également de la durée du congé rémunéré. Au Royaume Uni par exemple, le congé maternité n'est rémunéré de façon satisfaisante que les 6 premières semaines, la compensation est ensuite inférieure à $80 \%$ du salaire antérieur.

La rémunération concerne également le congé parental. On constate alors des variations très importantes d'un pays à l'autre, quant au type de rémunération et à son montant. Ainsi, il n'est pas rémunéré aux Pays Bas, en Grèce, au Portugal, au Royaume Uni, en Espagne et en Irlande. Il existe par ailleurs des prestations forfaitaires en France, au Luxembourg, en Slovaquie et en France par exemple. La rémunération peut également correspondre à une compensation proportionnelle à la perte de salaire. C'est le cas en Italie, en Hongrie, en Finlande et en Suède. Mais le pourcentage de la compensation varie : de $30 \%$ en Italie, à $80 \%$ du salaire en Suède.

C'est le croisement de ces deux éléments, durée et rémunération des congés, qui permet de comprendre les possibilités de conciliation offertes aux familles et l'impact des différents dispositifs sur l'égalité hommes - femmes. Antoine Math et Christèle Meilland distingue ainsi quatre groupes de pays (2004b) :

Groupe 1 : le Royaume-Uni, les Pays-Bas, le Portugal, l'Irlande et la Grèce où le droit à un congé parental est faible. Il est en effet faiblement rémunéré et d'une durée réduite. Il faut mentionner au Royaume Uni l'existence d'un congé de maternité, donc réservée aux mères, d'une durée de 52 semaines, mais dont la moitié seulement est rémunéré. 
Groupe 2 : la Belgique, le Luxembourg et l'Italie. Comme dans le groupe 1, les congés sont de courte durée, mais ils sont mieux rémunérés.

Groupe 3 : L'Autriche, l'Allemagne, la Hongrie, la Slovaquie, la France et la Pologne, où le congé parental, d'une durée de 2 à 3 ans, rémunéré sous la forme d'une prestation forfaitaire (sauf en Hongrie) ${ }^{10}$.

Groupe 4 : le Danemark, la Finlande, la Norvège et la Suède qui se distinguent des autres pays car ils proposent un congé plutôt bien rémunéré (compensation proportionnelle au revenu d'activité), d'une durée limitée et qui concerne les deux parents.

D’une manière générale, prendre un congé parental, même lorsqu'il s'appuie sur un dispositif visant à renforcer l'égalité hommes - femmes, reste le fait des femmes. Comme le constate la littérature féministe, la conciliation vie familiale - vie professionnelle permet aux femmes d'articuler vie familiale et vie professionnelle, mais les mesures mises en œuvre ne remettent pas en question l'inégale répartition des tâches au sein de la sphère privée. Les taux d'activité des femmes ont certes augmenté dans les pays européens, mais l'organisation des tâches entre les hommes et les femmes n'a pas évolué de façon significative (Périvier, 2004). Le care demeure principalement une affaire de femmes. La faible rémunération des congés parentaux, les inégalités salariales entre les hommes et les femmes et la prégnance des normes familiales traditionnelles expliquent cette situation. Ainsi, un congé parental de longue durée, mais rémunéré faiblement (groupe 3) sera plutôt pris par les femmes et entraînera des difficultés de réinsertion sur le marché du travail. A l'inverse, la mise en place d'un congé parental de courte durée (groupes 1 et 2) évite l'éloignement durable du marché du travail, mais il pose d'autres problèmes de conciliation, liés à l'organisation de la garde des enfants. Une politique de ce type suppose donc de développer l'offre de garde, comme l'explique Monique Kremer dans cet ouvrage à propos du Royaume Uni. L'instauration dans certains pays d'un congé paternité ou de dispositifs comme les «quotas des pères » incitant les hommes à utiliser leur congé et à s'impliquer plus activement dans les tâches de care constituent une évolution significative mais encore insuffisante vers l'égalité hommes - femmes.

L'analyse des différentes formes de congés maternité, paternité ou parentaux montre qu'il existe d'importantes disparités entre les pays de l'Union Européenne (voir tableau 5). Ces différences, qui perdurent même si les mesures prises par l'Union européennes ont permis certains ajustements, témoignent de la diversité des régulations politiques de chacun des pays

\footnotetext{
${ }^{10}$ Antoine Math et Christèle Meilland incluent dans ce groupe l'Espagne où la durée du congé est également longue mais non rémunéré.
} 
et des différences des représentations de la répartition du travail rémunéré et non rémunéré au sein du couple. On le constate au niveau des politiques nationales comme des politiques européennes, l'enjeu de l'égalité hommes - femmes ne se pose pas seulement dans la sphère professionnelle, mais également dans la sphère privée. En d'autres termes, qui, au sein du couple prend le congé parental ? En quoi le type de congé adopté encourage-t-il ou non l'égalité au sein du couple et par là même la conciliation vie familiale - vie professionnelle ? Les dispositifs existant donnent ils aux parents le choix de travailler et/ou de s'occuper de leur enfant?

Tableau 5 : Données sur les congés parentaux (source : Silvera, 2007)

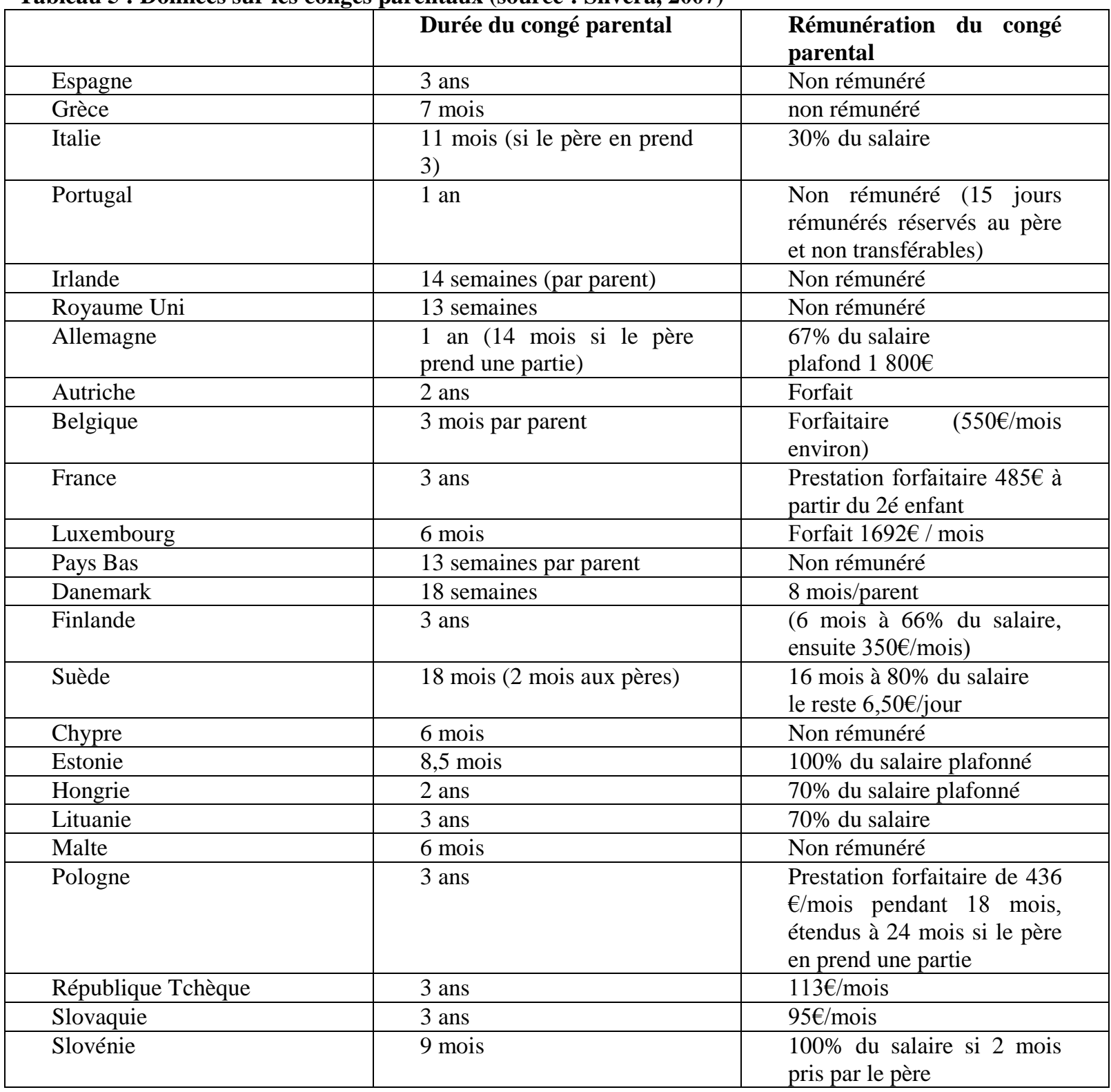




\section{La controverse sur "le libre choix »}

Les instruments des politiques de conciliation vie familiale - vie professionnelle sont donc variés : promotion du temps partiel pour les femmes, réduction et régulation du temps de travail, développement de services à la petite enfance, et en particulier de services publics, définition de formules de congé parental plus ou moins long, mais aussi plus ou moins bien rémunéré. Cette diversité d'instruments est la plupart du temps conçue comme devant faciliter « le libre choix » des parents, et surtout des mères. Grâce à cette palette de solutions, celles-ci devraient, par hypothèse, pouvoir choisir et ajuster au fil du temps, de la croissance des enfants et de leurs aspirations, les solutions pour mieux articuler leur activité professionnelle et leurs responsabilités parentales. Une mère pourrait ainsi prolonger son congé de maternité par un congé parental rémunéré avant le premier ou le deuxième anniversaire de son enfant, voire en bénéficier à temps partiel pour ne pas se couper de son activité professionnelle, puis le confier à des dispositifs de prise en charge combinant ressources formelles (crèches, assistantes maternelles, école maternelle) et informelles (réseau de proches, parents et/ou amis), jusqu'à son accès à l'école primaire. Bien sûr, ce tableau idyllique du « libre choix » ne tient pas compte des contraintes qui s'imposent inégalement dans la réalité : impossibilité d'accéder au marché du travail pour les uns, impossibilité de se passer d'un salaire pour les autres, absence d'offres de garde formelle ici ou de soutien informel, là, etc.

Cette rhétorique du «libre choix » est particulièrement développée dans le cas français. Mais elle fait écho également à la théorie des «préférences » des mères, dont Catherine Hakim, de la London School of Economics (2000, 2003 et 2004) est la représentante la plus connue. Cet auteur défend dans une série de publications basée sur des analyses statistiques sur plusieurs cas nationaux (Royaume-Uni, Espagne, Belgique, Allemagne, Suède, République Tchèque et Japon) que l'on peut repérer plusieurs catégories de femmes, du point de vue de leur relation au travail et à l'emploi; femmes qui concevraient donc de manière très contrastée l'articulation entre vie familiale et vie professionnelle. Celles-ci manifesteraient donc trois types de préférences conduisant à trois principaux modes de vie :

- une première catégorie de femmes (qui représente entre 10 et $30 \%$ des cas, selon les pays) donnerait la priorité à leur vie de famille et à leurs enfants et ferait le choix de ne pas s'inscrire sur le marché du travail pour satisfaire cette préférence. Ces femmes privilégieraient des valeurs comme le souci de l'autre, le partage, le refus de la compétition ; 
- une deuxième catégorie (également entre 10 et 30\% des cas, selon les pays) ferait au contraire le choix de la carrière, de l'investissement professionnel ou dans des activités de la sphère publique, en ayant recours aux valeurs de compétition, de concurrence, de réalisation individuelle et d'excellence. C'est dans cette catégorie que l'on trouve le plus fréquemment les femmes qui renoncent à avoir des enfants.

- Enfin, une troisième catégorie intermédiaire en quelque sorte, et représentant entre 40 et $80 \%$ des cas selon les pays, regrouperait les femmes qui font des compromis entre réalisation personnelle, familiale et professionnelle. Elles désirent travailler, mais refusent d'être complètement déterminées par les obligations de performance, qu'impose le monde du travail. Cette catégorie serait donc pas hypothèse la plus sensible aux politiques publiques de conciliation, qui leur permettrait de faire ces compromis entre engagement professionnel et réalisation au sein de leur vie privée.

Cette typologie est conçue par l'auteur comme une « evidence-based theory » (Hakim, 2007), directement issue de regroupements statistiques et sans a priori normatif. Elle ne dit rien cependant sur le fait que ces catégories soient aussi inégales en proportion, au point que la catégorie intermédiaire capte jusqu'à près de $80 \%$ des supposés profils de préférences. Mais l'idée défendue qui nous importe ici est que, dans nos sociétés individualisées, certaines femmes préféreraient spontanément et invariablement le rôle de «mère au foyer », quand d'autres, au contraire, privilégieraient en quelque sorte la carrière.

Pour Hakim, les préférences personnelles sont de moins en moins déterminées par les normes sociales, dans la mesure où les individus ont plus que jamais la possibilité de s'en émanciper, de définir leurs comportements par rapport à eux-mêmes, d'où l'idée de préférences, justement ${ }^{11}$. Sa théorie fait donc également écho aux thèses sur l'individualisation qui caractériserait les sociétés post-industrielles de la seconde modernité (Giddens, 1992 ; Beck, 1992); des sociétés réflexives où chaque individu chercherait à écrire sa propre histoire, composer sa propre trajectoire, en posant à tout moment des choix. Cette aspiration à l'autodétermination serait le résultat du processus de démocratisation, aussi bien dans la sphère publique que privée (Commaille et Martin, 1998 et 1999).

\footnotetext{
${ }^{11}$. Comme elle l'écrit elle-même : «Le lien entre les normes sociétales et les préférences personnelles est faible. (On peut être d'accord avec le fait que c'est une bonne idée pour tout le monde d'arrêter de fumer et choisir cependant de fumer soi-même). Les normes sociétales sont des attitudes non-causales ; elles font partie du patrimoine social, mais ne prédisent pas le comportement individuel. Les préférences personnelles sont des attitudes causales et forgent fortement les comportements » (2007, p. 127).
} 
Mais la thèse des préférences prend de toute évidence le risque de minorer les conditions de possibilités du choix, autrement dit le poids des contraintes inégales qui s'exercent sur les individus selon leur appartenance sociale. Pour choisir, il faut indéniablement avoir un certain nombre de ressources. Autrement, le choix peut n'être qu'une rationalisation a posteriori d'un non-choix ou d'une absence d'alternative. Car, en effet, les sociétés contemporaines sont peut-être des sociétés qui promettent l'auto-détermination, mais ce sont aussi des sociétés terriblement inégalitaires qui suscitent donc beaucoup de déceptions, de frustrations liées à des promesses non tenues, ce que souligne Beck lui-même dans certains de ses derniers écrits (Beck et Lau, 2005).

Là réside précisément la controverse engagée avec de nombreux auteurs (voir par exemple Crompton et Lyonette, 2005 et 2007). Peut-on en effet sans plus de précautions considérer que les femmes ont désormais, dans les sociétés développées, le choix de composer leurs trajectoires de vie, que ce soit celui d'avoir ou non des enfants (grâce à la révolution contraceptive) ou celui de travailler ou non ? Derrière cette controverse, on retrouve la question fondamentale pour la sociologie des déterminations sociales, du poids des contraintes et des inégalités. Pour Rosemary Crompton et Clare Lyonette, la théorie des préférences s'apparente à la vision donnée de la vie familiale par l'économie néo-classique et la théorie du choix rationnel. Mais pour ces auteurs, Catherine Hakim n'est pas loin non plus de la posture féministe essentialiste, qui voit dans la nature des femmes (ou de certaines femmes, peut-être) les fondements de leur différence et se refuse à toute analyse des rôles des sexes en termes de construction sociale; pas si loin non plus d'un féminisme conservateur et populiste qui défend que les femmes vivant en couple sont plus « heureuses » si elles adoptent une vision traditionnelle des rôles des sexes dans la sphère domestique.

La deuxième partie de cet ouvrage est consacrée à cette discussion de la théorie des préférences. Sans se limiter à la controverse idéologique, les trois chapitres de cette partie mettent à l'épreuve et discute le modèle de Catherine Hakim et en montrent les limites, en ayant recours pour deux d'entre eux (Olli Kangas et Tine Roostgaard ; Mara Yerkes) à des méthodes statistiques comparables à celles de Hakim. Monique Kremer défend pour sa part l'idée qu'un certain nombre de variables culturelles interagissent pour composer des « idéaux de care », un système complexe de représentations collectives, qui font que telle ou telle modalité de conciliation apparaît recevable dans un contexte national et historique donné.

A la lumière de cette partie de l'ouvrage, on pourra en tous les cas constater à quel point cette notion de «libre choix » pose problème et nécessite de plus amples recherches. Mais il ressort 
des analyses proposées qu'il est sans aucun doute nécessaire de mettre en regard les discours des acteurs (hommes et femmes), leurs aspirations, leurs souhaits, que ce soit sur leur relation au travail et à l'emploi, leur rôle de parent, et leurs pratiques de conciliation vie familiale vie professionnelle avec un contexte qui comprend aussi bien une offre de services (des politiques sociales) et des «idéaux de care » (modèles culturels).

\section{Dégradation de la qualité des emplois féminins et difficultés de conciliation}

Parce que la question de la conciliation vie familiale - vie professionnelle ne se développe pas dans un vide sociétal, cet ouvrage se propose aussi de faire le point sur la manière dont se pose concrètement ce problème dans tel ou tel secteur d'activité, en tenant compte des évolutions en cours du marché du travail. C'est ce que proposent Paul Bouffartigue et Jacques Bouteiller en s'intéressant aux infirmières et aux femmes cadres dans le secteur bancaire dans trois pays (France, Belgique et Espagne), ou Nathalie Lapeyre en étudiant le cas des femmes exerçant une profession libérale, ou encore Anne-Eydoux et Marie-Thérèse Letablier en analysant l'évolution des pratiques de garde dans le secteur de la petite enfance pour répondre à de nouveaux besoins, comme les horaires atypiques. Ces professionnels de la petite enfance voient ainsi leurs conditions de travail se complexifier, voire se dégrader, puisqu'elles doivent elles aussi s'adapter à cette évolution des horaires de travail dans un ensemble d'autres secteurs. Le constat que dressent Diane-Gabrielle Tremblay, Renaud Paquet et Elmustapha Najem sur le Québec n'est guère différent. Là encore, l'écart se creuse entre aspirations des individus et conditions de travail.

De ce point de vue, le cas français est particulièrement intéressant. En effet, le débat sur la question de l'équilibre entre vie familiale et vie professionnelle s'y développe dans un contexte relativement paradoxal. Dans ce pays où le niveau de fécondité est le plus élevé d'Europe (près de deux enfants par femme en 2006), où près de $60 \%$ des femmes de 15 à 64 ans occupent un emploi, majoritairement à temps plein, où les services à la petite enfance et les écoles maternelles offrent une large palette de solutions de garde, le niveau de stress et la pression éprouvés par les parents pour articuler vie familiale et vie professionnelle restent élevés, surtout si on les compare à ce qu'il sont dans d'autres pays européens, comme le montre une récente enquête comparative menée par Rosemary Crompton (2006). A la lumière 
de ces résultats, ce que l'auteur appelle les work - life conflicts repérés dans les ménages (enquête ISSP) ou encore le stress lié à la work - life balance sont aussi importants en France qu'ils le sont au Royaume-Uni, certes moins importants qu'aux Etats-Unis voire au Portugal, mais sensiblement plus qu'en Norvège ou en Finlande (Crompton, 2006, p. 132).

Pour expliquer ce paradoxe, Crompton insiste sur le facteur suivant : la très inégale répartition des tâches domestiques et de soins entre les genres, ce qu'elle qualifie de «gender traditionalism $»^{12}$. Hélène Périvier défend une position assez voisine lorsqu'elle évoque pour sa part à propos du cas français : «un statu quo inégalitaire » (Périvier, 2007, p.76), c'est-àdire l'acceptation silencieuse d'une responsabilité principalement féminine de ces enjeux de conciliation (voir aussi Méda et Périvier, 2007).

Cette hypothèse d'un traditionalisme, d'un conservatisme, voire d'un familialisme français mériterait certainement de plus amples développements. Dans cet ouvrage, Amélie Benoît, pour le Québec, Danièle Boyer et Jeanne Fagnani pour la France analysent les évolutions en cours du côté des pères.

Mais on peut avancer un autre facteur pour expliquer cette tension et ces difficultés de conciliation dans les ménages français : la situation du marché du travail et sa dégradation au cours des dernières décennies. Comme l'indique Pierre Concialdi : «Depuis le début des années 1980, les emplois temporaires ou à temps partiel ont fortement augmenté : multiplication par cinq pour l'intérim, par quatre pour les CDD, par plus de trois pour les stages et contrats aidés et doublement de la population en sous-emploi. Au total, le volume des emplois précaires a augmenté de 2,5 millions entre 1983 et 2005, cette hausse absorbant plus de $60 \%$ de la croissance totale de l'emploi salarié (4 millions) » (Concialdi, 2006, p. 20). Si l'on considère la situation en 2003, les emplois précaires concernaient environ 4 millions de personnes en additionnant les personnes en sous-emploi, à temps partiel, occupant des emplois temporaires, à durée déterminée, les apprentis, les emplois aidés et les personnes en stage. Et si l'on y ajoute les chômeurs, le «précariat », au sens que lui donne Robert Castel (2006), pourrait concerner 6,5 millions de personnes. Et à cette masse considérable, on peut encore ajouter les chômeurs «découragés » (760 000 personnes, dont 56\% de femmes en 2001), les chômeurs indisponibles qui ne peuvent occuper immédiatement un emploi le plus souvent à cause de leurs responsabilités parentales (220 000, dont $67 \%$ de femmes) et les chômeurs qui ont travaillé plus d'une heure au cours de la semaine précédant l'enquête, ce qui

\footnotetext{
12 . "Malgré une longue histoire d'intervention publique pour soutenir le travail des femmes et dispenser des prestations aux familles, à la fois les stéréotypes de genre en matière d'emploi et de division du travail domestique sont plus conventionnels (ou traditionnels) en France que l'on aurait pu s'y attendre. (Crompton, 2006, p. 132).
} 
les exclut des statistiques du chômage (994000 personnes, dont 60\% de femmes). Ces chiffres montrent non seulement l'ampleur de cette précarité, mais aussi l'importance des inégalités de genre (Maruani, 2005 ; voir aussi Paugam, 2000).

En comparant les résultats des enquêtes emploi en France en 1993 et en 2002, et à partir d'une typologie des emplois occupés (en termes de statuts des emplois, de temps de travail et d'aménagement des horaires), Séverine Lemière et Céline Marc (2005) mettent clairement en évidence cette relative dégradation des conditions d'emploi des femmes au cours de la période. En 2002, près de $40 \%$ des femmes occupaient des emplois dits atypiques (intérim, CDD à horaires atypiques, contrats précaires, emplois aidés, travail à temps partiel choisi et subi), contre seulement $13 \%$ des hommes. Et donc a contrario, «les femmes sont sousreprésentées dans l'ensemble des classes à statut d'emploi typique ; elles sont moins présentes dans la classe des emplois associant statut typique et horaires 'normaux'. Or cette classe serait la mieux adaptée à l'articulation entre vie familiale et vie professionnelle et l'accès aux modes de garde des enfants. De plus, les femmes sont très fortement surreprésentées dans la classe associant contrat atypique de travail, principalement à temps partiel, horaires variables, travail du week-end et bas salaire ». Le nombre de ces emplois à statut atypique a sensiblement augmenté entre 1993 et 2002. «Les classes à statut atypique regroupaient 19,2\% des salariés en 1993 pour atteindre 24,7\% des salariés en $2002 »$.

A l'appui des données de la DARES, Florence Jany-Catrice (2006) souligne également l'importante progression du nombre des emplois non qualifiés depuis le début des années 1990 (5,7\% de progression annuelle entre 1994 et 2002). En 2003, ces emplois occupés à 80\% par des femmes, dont la moitié à temps partiel, le plus souvent contraint, concernent 5,3 millions de personnes. Ces emplois féminins non qualifiés sont non seulement faiblement rémunérés (en 2002, 40\% perçoivent des rémunérations inférieures à la moitié du salaire médian), mais s'exercent aussi souvent avec des horaires morcelés, fragmentés. Il n'est donc guère étonnant que la question de l'articulation vie familiale - vie professionnelle pose problème dans un nombre non négligeable de ménages français.

A partir de l'enquête «histoires de vie et construction des identités » de l'INSEE, Hélène Garner, Dominique Méda et Claudia Senik ont tenté d'évaluer ces difficultés de conciliation. Près de $40 \%$ des actifs occupés considèrent que « leur travail rend difficile l'organisation de leur vie de famille », et ce d'autant plus qu'ils ont des enfants et, tout particulièrement de jeunes enfants. Ainsi «la moitié des parents trouvent difficile de tout concilier quand ils ont, dans leur foyer, des enfants de moins de 11 ans »(Garner et al, 2004). Plusieurs autres 
variables influencent cette perception : le nombre des enfants, l'âge des parents, mais aussi le secteur d'activité, le statut des emplois et l'organisation des horaires de travail. $64 \%$ des hommes et $60 \%$ des femmes exerçant une profession appartenant à la catégorie des « artisans, commerçants et chefs d'entreprise », mais aussi $46 \%$ des femmes appartenant à la CSP «cadres et professions intellectuelles supérieures » ont le sentiment que la conciliation est difficile.

Les horaires de travail joue un rôle important. «Plus les horaires sont atypiques, plus les actifs trouvent qu'il est difficile de concilier vie familiale et vie professionnelle » (ibid). C'est le cas pour $80 \%$ des mères dont les horaires de travail sont alternants (par rapport à $40 \%$ des pères), mais aussi de $72 \%$ des mères (et de $68 \%$ des pères) qui travaillent de nuit plus d'une fois par semaine ; ou encore de $67 \%$ des mères (et $64 \%$ des pères) qui travaillent le dimanche et les jours fériés; et de $62 \%$ des mères (et $60 \%$ des pères) qui travaillent avant 7 heures le matin ou après $20 \mathrm{~h}$. Le lien entre statut de l'emploi, d'une part, imprévisibilité et irrégularité des horaires de travail, d'autre part, est complexe. Le fait d'occuper un emploi à durée déterminée à temps plein ou à temps partiel ne signifie pas nécessairement que ces personnes seront soumises à de telles contraintes. En revanche, les intérimaires sont manifestement les plus mal lotis ${ }^{13}$.

On le voit, il est difficile de dresser une cartographie exhaustive des conditions concrètes de la conciliation vie familiale - vie professionnelle. Nous disposons cependant d'un certain nombre d'enquêtes représentatives pour en approcher la configuration, sans rien manquer des nuances qu'impose une telle analyse, en fonction des configurations familiales, des secteurs d'activité, des différences d'emploi selon le genre, etc. Quatre chapitres de cet ouvrage proposent des bilans de ces enquêtes et de ce que nous pouvons y apprendre. Le chapitre rédigé par Laurent Lesnard fait le point sur la ressource importante que représentent les enquêtes «budget - temps » de l'INSEE pour appréhender l'articulation famille - travail pour les couples biactifs avec de jeunes enfants. François-Xavier Devetter se propose plutôt d'analyser le choc entre le temps professionnel des parents et le temps des enfants en s'appuyant sur une enquête qualitative originale, puisqu'elle aborde, ce qui est assez rare pour être souligné, le point de vue des enfants sur les contraintes professionnelles de leurs parents. Dans notre contribution à la dernière partie, nous proposons pour notre part de tirer quelques leçons de l'enquête «modes de garde et d'accueil des jeunes enfants » réalisés par le

\footnotetext{
${ }^{13}$. « $48 \%$ des intérimaires déclarent ne pas avoir des horaires identiques d'un mois sur l'autre, contre $28 \%$ des CDI à temps complet ». Les intérimaires subissent des temps de travail peu prévisibles : «ils sont $30 \%$ à déclarer une 'mauvaise' ou 'très mauvaise' prévisibilité de leurs horaires et calendriers » (Cottrel et al, 2002, p. 175).
} 
Direction de la recherche, des études, de l'évaluation et des statistiques (DREES) du ministère de la Solidarité et de l'Emploi, sur la manière dont les familles résolvent en France la question de l'accueil et de la garde de leurs enfants hors des plages horaires standard des services à la petite enfance. Enfin Teppo Kröger revient à la comparaison européenne en s'intéressant à des ménages particulièrement vulnérables de ce point de vue, les familles monoparentales. L'étude qualitative comparée de ces situations dans cinq pays européens révèle que, malgré les écarts culturels, politiques et économiques entre les situations nationales, un certain nombre de profils de situations se retrouvent de manière invariable partout et démontrent l'importance d'une analyse «par le bas », qualitative et concrètes, des arrangements ou pratiques de conciliation ;

La compréhension de ce défi que représente pour l'Europe et pour les systèmes de protection sociale la conciliation vie familiale - vie professionnelle est à ce prix : démultiplier les angles, les problématiques et les matériaux empiriques. Nous espérons que cet ouvrage aura contribué à en améliorer la lecture et l'intelligibilité.

\section{Bibliographie}

Beck U., 1992. Risk Society. Towards a New Modernity. Londres, Sage.

Beck U., Lau C., 2005. "Second modernity as a research agenda: theoretical and empirical explorations in the 'meta-change' of modern society", The British Journal of Sociology, vol. 56,4 , pp. $525-557$

Castel, R. (2006), “Au-delà du salariat ou en deçà de l'emploi? L'institutionnalisation du précariat », in S. Paugam (ed), Repenser la solidarité. L'apport des sciences sociales. Paris, PUF, p. 415-433.

Commaille J. \& Martin C., 1998. Les enjeux politiques de la famille, Paris, Bayard.

Commaille J., Martin C., 1999. «Les conditions d'une démocratisation de la vie privée », in D. Borrillo, E. Fassin et M. Iacub (sous la dir.), Au-delà du Pacs. L'expertise familiale à l'épreuve de l'homosexualité. Paris, PUF, 'Politiques d'aujourd'hui', 1999 (2 2 ème édition 2001), p. 61-78. 
Concialdi P. (2006), «La France précarisée : un état des lieux », in M. Husson (ed), Travail flexible, salariés jetables. Fausses questions et vrais enjeux de la lutte contre le chômage. Paris, La découverte, p. 17-27.

Cottrell M., Letremy P., Macaire S., Meilland C., Michon F. (2002), « Le temps de travail des formes particulières d'emploi », Economie et statistiques, n³52-353, p. 169-189.

Crompton R., Lyonette C., 2005. "The New Gender Essentialism: Domestic and Family 'Choices' and their Relation to Attitudes", The British Journal of Sociology, 56 (4): 601-620.

Crompton R. 2006. Employment and the Family. The Reconfiguration of Work and Family Life in Contemporary Societies. Cambridge, Cambridge University Press.

Crompton R., Lyonette C., 2007. « Reply to Hakim », The British Journal of Sociology, vol. 58, 1, p. 133-134.

Conseil d'analyse économique (2007), Rapport sur le service public de la petite enfance. Département 'Questions sociales' (www.strategie.Gouv.fr )

Esping-Andersen G. (2002), «A Child-Centred Social Investment Strategy", in G. EspingAndersen, D. Gallie, A. Hemerijck, J. Myles, Why we Need a New Welfare State, Oxford, Oxford University Press, pp. 26-67.

Esping-Andersen G., avec B. Palier. 2008. Trois leçons sur l'Etat-providence. Paris. Le Seuil, "La République des idées".

Garner H., Méda D., Senik C. (2004), ’La difficile conciliation entre vie professionnelle et vie familiale", Premières informations, premières synthèses, n50-3.

Giddens A., 1992. The Transformation of Intimacy. Sexuality, Love and Eroticism in Modern Societies. Cambridge, Polity Press.

Hakim C., 2000. Work Life-style Choices in the $21^{\text {st }}$ Century: Preference Theory. Oxford: Oxford University Press.

Hakim C., 2003. Models of the Family in Modern Societies: Ideals and realities. Aldershot: Ashgate.

Hakim C., 2004. Key Issues in Women's Work: Female Diversity and the Polarisation of Women's Employment. London: Glasshouse Press.

Hakim C., 2007. «Dancing with the Devil ? Essentialism and other feminist Heresies », The British Journal of Sociology, vol. 58, 1, p. 123-132. 
Hantrais L., 2000. Gendered Policies in Europe. Reconciling Employment and Family Life. London: MacMillan Press.

Jany-Catrice F., 2006, «Emplois tertiaires: la précarisation du travail féminin », dans L. Maurin et P. Savidan (dir.), L'état des inégalités sociales en France. Observatoire des inégalités. Paris, Belin, pp. 151-154.

Jönsson A. et Perrier G., 2007. «Les politiques de conciliation de l'Union Européenne : dépassement ou déplacement de la dichotomie genrée public-privée ?», Journée d'études de la Formation doctorale appliquée de Science-Po Paris Genre et action publique, 8 juin 2007.

Kremer M., 2007. How Welfare States Care? Culture, Gender and Parenting in Europe. Amsterdam University Press.

Lemière S., Marc, C. (2005), «Qualité des emplois et genre : les effets croisés des statuts et des horaires de travail», Texte de travail, Matisse, http://matisse.univparis1.fr/fr/IMG/pdf/ES_lemière_et_Marc.pdf

Lewis J., 1997. «Gender and Welfare Regimes: Further Thoughts », Social Politics, summer, p. $161-177$.

Lewis J., 2006. “Quels moyens pour promouvoir quelle égalité des sexes? Les inégalités hommes-femmes dans la division du travail rémunéré et non rémunéré », Revue Française des Affaires Sociales, $\mathrm{n}^{\circ} 1, \mathrm{p}$. 161-185.

Martin C., 2002. «Des politiques familiales à la politisation de la vie privée en Europe », Informations sociales, $\mathrm{n}^{\circ} 102$, p. 16-25.

Martin C., 2003. «L'accueil des jeunes enfants en Europe. Quelles leçons pour le cas français ? » in F. Leprince, L'accueil des jeunes enfants en France : Etat des lieux et pistes d'amélioration, Paris, Publication du Haut Conseil de la Population et de la Famille, La Documentation française, p. 137-192.

Martin C., 2004. «Les politiques de l'enfance en Europe», in F. de Singly (dir), Enfants, adultes : vers une égalité de statuts ? Paris, éditions Universalis, pp. 171-182.

Maruani M. (dir). 2005. Femme, genre et sociétés. L'état des savoirs. Paris, La Découverte.

Math A. et Meilland C., 2004a. «Un état des lieux des congés destinés aux parents dans vingt pays européens », Revue de l'IRES, n6, p. 113-136. 
Math A. et Meilland C., 2004b. «Les congés aux parents : contre l'égalité entre femmes et hommes ?», Revue de l'IRES, n46, p. 137-165.

Méda D., Périvier H., (2007), Le deuxième âge de l'émancipation. La société, les femmes et l'emploi. Paris, Le Seuil, «La République des idées ».

OCDE, 2001 et 2006. Petite enfance, grands défis : Education et structures d'accueil. Paris, Publication de l'OCDE.

Paugam S. 2000. Le salarié de la précarité. Paris, PUF.

Pécresse V. (2007), Mieux articuler vie familiale et vie professionnelle. Rapport au Premier ministre. Paris, La Documentation française.

Périvier H., 2004. «Emploi des mères et garde des jeunes enfants en Europe, Revue de l'OFCE, juillet, p. 90-258.

Périvier H. (2007), «L'emploi des femmes ou le statu quo inégalitaire », in Alternatives économiques, «Spécial élections 2007 », p. 75-77.

Silvera R., 2002. Articuler vie familiale et vie professionnelle en Europe. Un enjeu pour l'égalité, Paris, la documentation française.

Silvera R., 2007. «L'articulation entre vie professionnelle et vie privée : un enjeu majeur pour l'égalité entre les hommes et les femmes en Europe», Conférence «Reconciliation between work, family and personal life. New challenges for social partners and public policies, 12th July 2007.

Stratigaki M., 2004. "The cooptation of gender concepts in EU policies: the case of reconciliation of work and family", Social Politics, Volume 11, ${ }^{\circ}{ }^{1}$, Spring, p. 30-56. 